\title{
LA AUDIODESCRIPCIÓN EN EL DISCURSO MULTIMODAL EN ESPAÑOL: A PROPÓSITO DE LA SATURACIÓN DE INFORMACIÓN EN LA PELÍCULA LA MISIÓN
}

\author{
JUAN MANUEL ARCOS URRUTIA \\ Universidad Cardenal Herrera (España) \\ Centro de Investigación CySOC (UAL)
}

\section{INTRODUCCIÓN}

Una de las necesidades socio-comunicativas a las que suelen hacer referencia las personas con alguna enfermedad rara que se manifiestan en pérdida severa de visión, tales como la aniridia o la retinosis pigmentaria, es la de poder acceder mejor a los contenidos audiovisuales (cine y televisión, fundamentalmente). Existe un desconocimiento generalizado por parte de la sociedad sobre qué herramientas son utilizadas para hacer más accesibles los textos a las personas con alguna discapacidad.

La audiodescripción, en adelante $\mathrm{AD}$, no es una excepción en este sentido (Vázquez, 2008: 38). Básicamente, consiste en la incorporación, por parte de un narrador externo (audiodescriptor), de una pista de audio complementaria con la información que se considera relevante para que las personas con deficiencia visual 'accedan' lo mejor posible a las imágenes (fijas o en movimiento) que forman parte de un determinado discurso o de una determinada obra artística (pieza teatral, cuadro, monumentos o películas). Podríamos decir, siguiendo a Snyder (2005: 936), que se trata de la verbalización de lo visual o, desde otra perspectiva, que permite oír lo que no se puede ver.

Estamos, por tanto, en la adaptación de textos multimodales para que sean recibidos a través de un solo canal: el auditivo. El audio complementario se inserta en el espacio de tiempo existente entre los diálogos de los personajes. No hay, pues, solapamiento entre la descripción y las voces de esos personajes del documento original. Esa adaptación, tal y como indica Benecke (2004: 78), no puede hacerse sin tener en cuenta que no todos los narradores tienen la misma sensibilidad hacia el documento audiodescrito, ni tampoco todos los receptores parten del mismo grado de dis- 
capacidad visual. De hecho, es muy diferente, como él mismo recuerda, la persona ciega que tiene memoria visual de la persona que tiene esa discapacidad de nacimiento.

El inicio del interés por la $\mathrm{AD}$ como objeto de investigación se sitúa a mediados de la década de los setenta del siglo xx (Frazier, 1975). Ese interés se extendió a otros investigadores a partir de la década de los ochenta y noventa (Cejudo, 1991; Silverwood, 1992; Galisteo, 1994; Navarrete, 1997a, 1997b) y ha llegado hasta la actualidad (Piety, 2004; Benecke y Doch, 2004; Snyder, 2005; Doloughan y Rogers, 2005; Hyks, 2005; Ramael y Vercauteren, 2007, 2010; Braun, 2007, 2008; Salway, 2007a, 2007b; Vercauteren, 2012). En el ámbito hispánico, de igual forma, han ido apareciendo en los últimos años interesantes trabajos sobre la AD (Hernández-Bartolomé y Mendiluce-Cabrera, 2004; Orero, 2005, 2007; Jiménez Hurtado, 2007a, 2007b, 2010; Bourne y Jiménez Hurtado, 2007; Puigdoménech et al., 2010; Orero y Vilaró, 2012).

La dimensión práctica de la $\mathrm{AD}$ también ha ido avanzando, como es natural, a lo largo de los últimos treinta años. Ha aumentado el compromiso de empresas de comunicación y también de asociaciones e instituciones. En este sentido, podríamos mencionar, por ejemplo, la aparición de distintas normativas europeas que promueven el cumplimiento de unos parámetros básicos de calidad (ITC, 2000; Benecke y Dosch, 2004; AENOR, 2005; Orero, 2005; Vercauteren, 2007; Greening, 2010). Ahora bien, es evidente que la existencia de normativas no ha conllevado en todos los casos su estricto cumplimiento.

Sin duda, queda mucho trabajo por hacer en el campo de la AD. Así lo afirmaba Philip J. Piety, quien además aludía a la necesidad de estudiar con mayor detenimiento las fórmulas que podrían mejorar la efectividad del proceso. También se refería a la intensificación de técnicas de evaluación de las audiodescripciones (2004: 454). Desde nuestro punto de vista, la efectividad del proceso de AD tiene que ver, inicialmente, con qué se dice y cómo se dice; y también con la facilidad de comprensión de lo dicho, tanto desde un punto de vista cuantitativo como cualitativo. En este sentido, una de las tareas pendientes es la evaluación cuidadosa del grado de saturación de información existente en los textos audiodescritos. La Norma española UNE 153.020 del año 2005 mencionaba justamente la saturación del producto audiodescrito como un elemento importante para no causar cansancio en el oyente con discapacidad visual (AENOR, 2005: 7).

La finalidad del artículo será determinar el nivel de saturación presente en el contenido de la información audiodescrita, valorar si ese factor puede incidir, de alguna manera, sobre la comprensión de una obra audiovisual (Arcos, 2012) y proponer alternativas que pudieran mejorar el acceso a los contenidos audiodescritos. 


\section{SATURACiÓN DE INFORMACiÓN}

Cuando se dispone de mucha información o cuando se ofrece una información muy compleja que hay que procesar en poco tiempo podemos desembocar en lo que llamaríamos síndrome de fatiga informativa (Whitelaw, 2008: 175) o saturación de información (SI). La SI es un concepto que ha interesado a disciplinas muy diversas, como por ejemplo a la informática, a la economía o a la lingüística (Grice, 1975; Itami, 1977; Stanley, 2000, 2007; Rosenberg, 2003; Recanati, 2004, 2007; Blair, 2010). También ha tenido un largo recorrido en el ámbito de la semiótica visual (Sedlack, Showm y Keller, 2009: 45; Margariños, 2001) y de los estudios sobre la percepción (Kosslyn, 1994, 2005). En 1979, Enoch, Campos y Bedell hablaban de un síndrome de fatiga visual o de saturación visual, término que ha sido posteriormente utilizado también por otros investigadores (Neetens, 1984: 25; Regan y Neima, 1984: 673; Wildberger y Robert, 1988: 167). De igual forma, se puede hablar de una fatiga auditiva, que puede condicionar la comprensión de la información recibida (Chávez, 2006: 44).

\subsection{Nivel de saturación en el proceso de audiodescripción}

Proponemos identificar el nivel de saturación de la narración audiodescrita a partir de dos dimensiones: cualitativa y cuantitativa. La segunda será la que tendrá un mayor protagonismo a la hora de realizar esa identificación. La primera, la cualitativa, nos servirá como reguladora o como filtro de los datos procedentes del estudio cuantitativo. En cuanto a la dimensión cuantitativa, que conforma lo que denominamos patrón rítmico o principal, hace referencia a la interrelación de las siguientes categorías:

- $\quad$ (P-TAD/TPG) el porcentaje de tiempo consumido por el audiodescriptor (TAD) en relación al tiempo de la pausa general (TPG). En esta categoría hay que tener presente también el tiempo de la pausa interna (TPI). El tiempo de la audiodescripción real (TADR) será, pues, la suma entre el (TAD) y el (TPI).

- La velocidad de habla calculada a partir del número de palabras (NP) en relación con el número de palabras por segundo (PS) y con el número de palabras por minuto (PM) consumidos.

La dimensión cualitativa hace referencia a las siguientes categorías, que se materializan en lo que denominamos patrón de complejidad o secundario:

- $\quad$ Complejidad de información (COM), que se materializa en dos categorías: una primera que llamamos léxico-sintagmática, formada, 
principalmente, por la ambigüedad (AMB) y la disponibilidad (DIS). Una segunda categoría está formada por la complejidad de las acciones descritas, donde nos centraremos, principalmente, en los actos (ACP) y los estados (ESP) de los personajes. Cuando hablemos de acto nos estaremos refiriendo, sobre todo, a acciones de movimiento de los personajes en la pantalla. Cuando nos ocupemos de estados nos referiremos a emociones, sentimientos y reflexiones de los personajes. Somos conscientes de que existe una interrelación entre acto y estado, aunque siempre que sea posible diferenciaremos entre uno y otro. Cualquier acción de los personajes sería un acto.

Tanto en el patrón rítmico como en el patrón de complejidad, el concepto de relevancia será determinante (Sperber y Wilson, 1994). Tal y como aparece resumido en la tabla 1, proponemos distintos grados del porcentaje de tiempo consumido, de velocidad de habla ${ }^{1}$ y de cantidad de acciones.

TABla 1. Valores de las variables Porcentaje, Velocidad y Acciones

\begin{tabular}{|c|l|l|}
\hline Porcentaje (P-TAD/TPG) & Velocidad (PM) & Acciones (ACP) \\
\hline Muy Alto $(+80 \%)$ & Muy Rápida (+200) & Muy Alto (+4) \\
\hline Alto (61-80\%) & Rápida (181-200) & Alto (3-4) \\
\hline Normal (50-60\%) & Normal (160-180) & Normal (2) \\
\hline Bajo (49-20\%) & Lenta (140-159) & Bajo (1) \\
\hline Muy Bajo (-20\%) & Muy Lenta (-140) & Muy Bajo (0) \\
\hline
\end{tabular}

Interrelacionando estas tres variables y los distintos grados propuestos, hemos extraído cinco niveles de saturación, tal y como aparecen en la tabla 2. En esta tabla, sólo aparecen categorías simplificadas, porque al relacionar las tres variables tendríamos más de 100 posibilidades distintas.

${ }^{1}$ Desde la psicología del lenguaje y la comunicación se ha prestado especial atención a la velocidad del habla y su relación con la comprensión de los mensajes discursivos. Algunos autores colocan la barrera de la comprensión (Knapp, 1982: 307) en las 275 o 300 palabras por minuto, pero referida al género conversacional. Ellis y McClintock (1993: 80) sitúan la escucha efectiva entre 150/200 palabras por minuto, ya que la comprensión comenzaría a verse afectada a partir de las 200 palabras por minuto. En relación al género informativo en contextos radiofónicos, se suele hacer referencia a una media aconsejable de 160/170 palabras por minuto (Hills, 1987: 135; McLeish, 1978: 71; Cebrián Herreros, 1994: 407). 
TABLA 2. Nivel de saturación

\begin{tabular}{|l|l|l|l|}
\hline Nivel de saturación & Porcentaje (TAD/TPG) & Velocidad (PM) & Acciones (ACP) \\
\hline Hipersaturación & (Muy) Alto & (Muy) Rápida & (Muy) Alta \\
\hline Semisaturación & Normal-(Muy) Alto & $\begin{array}{l}\text { Normal- } \\
\text { (Muy) Rápida }\end{array}$ & $\begin{array}{l}\text { Normal- } \\
\text { (Muy) Alta }\end{array}$ \\
\hline Compensación & Normal & Normal & Normal \\
\hline Semicompensación & $\begin{array}{l}\text { Normal-(Muy) Alto- } \\
\text { (Muy) Bajo }\end{array}$ & $\begin{array}{l}\text { Normal- } \\
\text { (Muy) Rápida- } \\
\text { (Muy) Lenta }\end{array}$ & $\begin{array}{l}\text { Normal- } \\
\text { (Muy) Alta- } \\
\text { (Muy) Bajo }\end{array}$ \\
\hline
\end{tabular}

Nos centraremos en el valor prototípico de cada categoría. Así, la AD será Hipersaturada cuando las tres posibilidades estén entre las categorías (Muy) alto-(Muy) rápida. En segundo lugar, la AD será Semisaturada si en una categoría tenemos Normal combinada con valores (Muy) alto-(Muy) rápida. Tendremos una $\mathrm{AD}$ Compensada cuando los tres valores se encuentren dentro de la categoría Normal. En cuarto lugar, la AD será Semicompensada cuando tengamos valores de Normal en alguna categoría y (Muy) alto-rápida con (Muy) bajo-lento. Finalmente, será una AD Descompensada cuando los tres valores sean opuestos, es decir, (Muy) alto-(Muy) lento(Muy) Bajo con (Muy) bajo-(Muy) rápido-(Muy) alto.

\section{Corpus Seleccionado}

Para este trabajo, nos centraremos en la audiodescripción de textos cinematográficos, en los que la $\mathrm{AD}$ hace referencia, principalmente, a las acciones de los personajes, al vestuario, a las expresiones faciales, al lenguaje corporal, a la descripción de la ambientación o a la justificación de sonidos o efectos sonoros a los que de otra forma las personas ciegas o con baja visión no podrían acceder adecuadamente (Whitehead, 2005: 960).

Decidimos, en primer lugar, ceñirnos a una muestra de películas que hubieran seguido la Norma española UNE 153.020. Y, en segundo lugar, contar con fragmentos de distintos géneros y registros y, en la medida de lo posible, con estilos diferentes de audiodescripción. Un fragmento de la película La Misión (Roland Joffé, 1986) fue finalmente el elegido, ya que 
cumplía con las características que nos habíamos marcado. Más concretamente, el arranque, ya que es en esta fase en la que suele haber mayor necesidad de información que contextualice el espacio general en el que se desarrolla la acción o que caracterice a los personajes principales (sus rasgos faciales o su vestimenta, por ejemplo).

\section{Metodología}

La determinación del nivel de saturación del fragmento audiovisual se desarrolló en varias fases, tal y como indicamos a continuación:

1. Identificación de las unidades de audiodescripción (UA), entendidas como cada uno de los segmentos de información que el audiodescriptor incorpora durante la emisión de la película y que técnicamente recibe el nombre de 'bocadillo de información' (AENOR, 2005).

2. Medición de la pausa general ${ }^{2}$ y del tiempo de esa pausa consumido por la audiodescripción.

3. Extracción del número de palabras y de sílabas por segundo de cada una de las UA para pasar a calcular, a continuación, la velocidad de la locución, expresada en palabras por minuto.

4. Selección para nuestro análisis de aquellas UA que estén por encima de los 10 segundos de pausa general y estén formadas por 15 palabras o más.

5. Exclusión de los títulos de crédito, porque pensamos que no forman parte del núcleo propiamente dicho de la audiodescripción.

6. Segmentación del número de actos por cada UA.

7. Interrelación de los datos obtenidos en las fases anteriores para calcular el mencionado nivel de saturación. Sólo nos detendremos en las combinaciones en las que el P-TAD/TPG sea (Muy) alto, ya que será en este contexto en el que los destinatarios pueden tener más dificultades de comprensión.

8. Una vez extraídas las UA prototípicas, analizaremos el papel desempeñado por el orden de la información, la ambigüedad y la disponibilidad léxica en la AD.

9. La identificación de UA Hipersaturadas, Semisaturadas o Descompensadas en la versión original (V. O.) sería la primera fase del análisis. En un segundo momento, buscaríamos una versión alterna-

${ }^{2}$ Para la medición de las pausas hemos utilizado el programa Adobe Audition 3.o. 
tiva (V. A.) que coincidiese con la categoría de Compensación, tal y como era descrita anteriormente en la tabla 2, pero nuestro objetivo final será detenernos en aquellas UA que estén Hipersaturadas o Semisaturadas.

10. Valoraremos posibles procesos de ajuste tanto en la forma (tiempo de audiodescripción y velocidad) como en el contenido (léxico utilizado y estructura sintáctica elegida para describir estados y acciones). Esos procesos consisten, básicamente, en la supresión o condensación de información, el cambio o reordenación de contenidos o la adición de información. Más concretamente, nuestra intención se dirigirá, por ejemplo, a la supresión de solapamientos y repeticiones, al cambio de léxico o estructuras sintácticas que pudieran dificultar la comprensión o, en casos determinados, a la incorporación de nuevos contenidos que mejorasen la expresión o que enriqueciesen la $\mathrm{AD}$. Si los ajustes supusiesen un problema en lo que respecta al seguimiento adecuado de la trama o implicase la eliminación de información relevante, nuestra propuesta sería ofrecer una V.A. que aunque pudiera ser Descompensada parcialmente en los aspectos formales (tiempo y velocidad), salvaría siempre la transmisión de información considerada relevante en cada contexto (Van Dijk, 2008: 18) y que pudiera estar referida al qué o al quién, a lo que se hace o dice y a otras dimensiones complementarias relacionadas con el tiempo, el lugar, el modo o el motivo. En todo caso, habría que evitar que se produjese una descompensación absoluta, ya que interfiere claramente en la comprensión y en la recepción de los mensajes audiodescritos.

\section{Resultados}

El fragmento analizado de la película La Misión está formado por un total de 102 UA, de la cuales solamente 13 están por encima de 15 palabras y de 10 segundos de pausa general, que son las UA válidas para nuestro análisis, de las cuales, solamente 5 tienen un P-TAD/TPG (Muy) alto, es decir, un $38,46 \%$. El porcentaje, por tanto, es de 12,74\%, mientras que, por otro lado, aparecen 89 UA no válidas.

Observamos que, en el arranque de esta película, de las 13 UA válidas, existe una categoría del nivel de saturación que sobresale en comparación con el resto. Nos estamos refiriendo a la categoría de la Semisaturación (6 UA en total), ya que el ritmo es muy rápido en el inicio y el audiodescriptor no adecua muy bien el tiempo de la pausa general, así como su velocidad a la información audiodescrita. 
TABLA 3. Nivel de saturación en el fragmento analizado de La Misión

\begin{tabular}{|l|c|c|}
\hline Nivel de saturación & Número de UA & Porcentaje \\
\hline Semisaturación & 6 & 46 \\
\hline Semicompensación & 4 & 31 \\
\hline Hipersaturación & 3 & 23 \\
\hline Compensación & 0 & 0 \\
\hline Descompensación & 0 & 0 \\
\hline Total & $\mathbf{1 3}$ & $\mathbf{1 0 0}$ \\
\hline
\end{tabular}

A continuación, presentamos una tabla con los datos del patrón rítmico del fragmento audiovisual audiodescrito analizado. Hemos focalizado el análisis sólo en las UA en las que el porcentaje TAD/TPG está en los valores (Muy) alto.

Tabla 4. Datos del patrón rítmico de La Misión

\begin{tabular}{|c|c|c|c|c|c|c|c|c|}
\hline UA & TPG & TADR & TAD & TPI & $\%$ TAD/TPG & NP & PS & PM \\
\hline 26 & 14,339 & 12,357 & 11,301 & 1,056 & 78,81 & 42 & 2,99 & 179 \\
\hline 27 & 10,789 & 9,452 & 8,993 & 0,459 & 83,35 & 30 & 3,17 & 190 \\
\hline 37 & 15,169 & 9,959 & 7,469 & 2,490 & 65,65 & 37 & 2,71 & 162 \\
\hline 83 & 10,637 & 7,102 & 6,594 & 0,508 & 61,99 & 24 & 3,37 & 202 \\
\hline 84 & 10,743 & 7,546 & 7,162 & 0,384 & 66,67 & 30 & 6,22 & 238 \\
\hline
\end{tabular}

\section{ANÁlisis DE LOS DATOS}

Una vez que tenemos todos los resultados del patrón rítmico y del patrón de saturación, consideramos como UA prototípica aquella en la que la variable P-TAD/TPG está por encima del $75 \%$ y el TPG de más de 10 segundos. De este modo, tendríamos 2 UA.

Las UA no 26 y no 27 serán las que tomemos como referencia para la realización de una versión alternativa para presentar una AD compensada. Nuestra intención será, por tanto, evitar esa sensación de cansancio por saturación. 
Entre paréntesis aportamos el valor de la pausa interna porque creemos que tiene un valor estratégico discursivo, en ciertos casos. El audiodescritor, a partir de la focalización de alguna palabra con el uso de la pausa, quiere que el usuario ciego o con deficiencia visual tenga presente que esa palabra es relevante en ese momento preciso.

El porcentaje consumido por la $\mathrm{AD}$ en relación al tiempo total de la pausa de la UA n 26, "Director de fotografía, Chris Menges. Tres jesuitas recorren el cañón por cuyo fondo discurren las aguas procedentes de las cataratas (0.433). Uno de ellos es Fielding, interpretado por Liam Neeson (0.422). Otro es Gabriel (0.188), alto, delgado, moreno y con barba, interpretado por Jeremy Irons", está formado por un $78,81 \%$. Identificamos esta unidad audiodescrita como Hipersaturada.

Afirmamos que está saturada porque en un mismo bocadillo aparecen dos tipos de información audiodescrita; por un lado, la localización de la acción dramática y, por otra parte, la descripción de los personajes.

En relación a este segundo elemento, el audiodescriptor omite a uno de los tres jesuitas y solo se detiene en la descripción de dos de ellos. Este aspecto podría ser mejorable en una $\mathrm{AD}$ alternativa. Se produce otro hecho muy interesante, que hace referencia al orden que utiliza el audiodescriptor en la presentación de los personajes. Creemos que no es muy apropiado describir en primer lugar a Fielding y seguidamente a Gabriel, sino que tendría que ser justamente al revés por la importancia y relevancia que este personaje tiene a lo largo de la película. Finalmente, aparece en la AD el término "cañón", en la acepción no 10 del DRAE, es un "paso estrecho y o garganta profunda entre dos altas montañas, por donde suelen correr los ríos". Es un concepto que no queda muy claro, donde tenemos un ejemplo de la llamada disponibilidad léxica.

Para esta UA hemos optado por realizar dos versiones alternativas. La primera (V.A.1), "Director de fotografia Chris Menges (0.500). Tres jesuitas caminan por la orilla del río cerca de las cataratas (0.474). Son Gabriel, alto, delgado y de barba castaña, interpretado por Jeremy Irons (0.423), Fielding, interpretado por Liam Neeson y otro jesuita, canoso". Está formada por 38 palabras. Como primera intención para mejorar la $\mathrm{AD}$, hemos reducido un poco el número de palabras de la versión original. También hemos eliminado la expresión "recorren el cañón" porque creemos que muchos usuarios no van a entender correctamente este término. Así en nuestra versión hemos creído conveniente situar a los personajes en un lugar físico concreto, en este caso, " $c a$ minan por la orilla del río cerca de las cataratas". Hemos realizado una pausa de casi medio segundo, concretamente 0.474, para focalizar en el término cataratas, con el fin de situar al espectador. En este sentido, aparece la información relevante necesaria para seguir adecuadamente la trama. Por otro lado, hemos presentado a los personajes por su orden de relevancia a lo largo de la película, es decir, primero a Gabriel, seguidamente a Fielding 
y, finalmente, se identifica al religioso canoso. Pensamos, además, que Gabriel debe tener un grado de descripción mayor, dada su importancia a lo largo de la obra cinematográfica.

Por otro lado, hemos optado por la realización de otra versión alternativa (V.A.2) mucho más reducida, con un total de 31 palabras. Hemos decidido eliminar, asimismo, las descripciones físicas de las personas para evitar, en la medida de lo posible, esa sensación de cansancio por saturación. Por lo tanto, esta versión alternativa quedaría de la siguiente manera: "Director de fotografía Chris Menges (0.294). Tres jesuitas caminan por la orilla del río cerca de las cataratas (0.478). Son Gabriel, interpretado por Jeremy Irons, Fielding, interpretado por Liam Neeson y otro jesuita".

La UA no 27 de la $\mathrm{AD}$ original realizada por la ONCE dice así: "El río cae desde la altiplanicie por grandes cataratas en forma de herradura que cierran el valle (0.212) y discurre entre paredes cubiertas de vegetación (0.247) que ascienden verticales hasta la meseta".

El porcentaje consumido por la AD en relación al tiempo total de la pausa es del 83,35\%, con una velocidad de 190 ppm, es decir, estamos ante una UA Hipersaturada. Además, en la película aparece justamente seguida a la UA anterior. El ritmo, así como la velocidad, hacen que estemos ante una sensación de saturación elevada. En este bocadillo nos encontramos con una descripción muy exhaustiva del paisaje.

Hay que indicar también que el léxico empleado es muy complejo y no se llegaría a comprender muy bien la descripción, ya que estamos ante palabras muy complejas, desde el punto de vista de la disponibilidad léxica, por parte de la mayoría de espectadores o ante expresiones léxicas ambiguas. Nos estamos refiriendo a términos como "altiplanicie" o "en forma de herradura".

También aparece en esta UA una metáfora muy interesante que contribuye a ralentizar la comprensión de la escena cuando se mencionan las "paredes" por donde discurre el agua. Todas estas indicaciones lingüísticas sólo son filtros que se complementan entre sí con la categoría cuantitativa que da lugar a una UA saturada y, como consecuencia, el contenido audiodescrito dificulta parcialmente la comprensión de la escena.

La propuesta alternativa (V.A. ${ }_{1}$ ) que presentamos "Los tres jesuitas se detienen (0.200). Fielding señala hacia las grandes cataratas, revestidas de vegetación, por donde discurre el caudaloso río", está formada por 20 palabras.

En este sentido, hemos creído oportuno evitar la descripción del paisaje y detenernos exclusivamente en lo que hacen los personajes. En primer lugar, se detienen y, seguidamente, Fielding señala las cataratas. En nuestra versión alternativa hemos conseguido, por un lado, evitar el cansancio por saturación y, en segundo lugar, describir exactamente lo que hacen los personajes, sin entrar en la descripción del paisaje puesto que pensamos 
que no es muy relevante en esta UA. También hemos optado por presentar un léxico mucho más sencillo que el usuario ciego o con deficiencia visual comprenderá mucho mejor.

Hemos realizado otra versión audiodescrita (V.A.2): "Los tres jesuitas se detienen (0.171). Fielding señala las impresionantes cataratas, conformadas por abundante vegetación". Es una versión mucho más simplificada en cuanto al número de palabras, pero mantiene las acciones de los personajes. En este sentido, tenemos una UA de 14 palabras y también hemos reducido el grado de descripción de la ambientación por considerarla, como en la versión anterior, menos relevante.

Una vez que hemos realizado nuestras versiones alternativas, presentamos en la tabla 5 los valores del patrón rítmico. En primer lugar, decimos que, aunque no hemos podido equiparar los valores a una audiodescripción Compensada, hemos logrado que esa sensación de cansancio por saturación sea mucho menor y, como consecuencia, se llega a comprender más fácil la trama. Como vemos en la tabla, los valores de los porcentajes, salvo

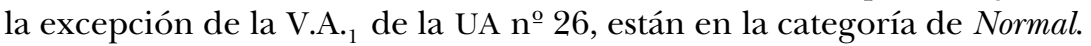

TABLA 5. Patrón rítmico de las versiones alternativas de La Misión

\begin{tabular}{|c|c|c|c|c|c|c|c|c|}
\hline UA & TPG & TADR & TAD & TPI & \% TAD/TPG & NP & PS & PM \\
\hline $26 \mathrm{~V}_{1}$ & 14,339 & 13,750 & 12,353 & 1,397 & 86,14 & 38 & 2,76 & 165 \\
\hline $26 \mathrm{~V}_{2}$ & 14,339 & 9,330 & 8,558 & 0,772 & 59,68 & 31 & 3,32 & 199 \\
\hline $27 \mathrm{~V}_{1}$ & 10,789 & 6,240 & 6,040 & 0,200 & 55,98 & 20 & 3,20 & 192 \\
\hline $27 \mathrm{~V}_{2}$ & 10,789 & 5,799 & 4,970 & 0,171 & 53,74 & 14 & 2,81 & 144 \\
\hline
\end{tabular}

\section{Conclusiones}

Este trabajo, por tanto, nos ha permitido llegar a las siguientes conclusiones:

a) Hay una escasez de trabajos en español que analicen la $\mathrm{AD}$ y la saturación, por lo que sería conveniente intensificar este tipo de investigaciones dado el valor estratégico que tiene el tiempo de la pausa en este modo de accesibilidad en los medios audiovisuales.

b) Hemos presentado una primera aproximación del estudio del patrón rítmico y su relación con el nivel de saturación en las películas audiodescritas. 
c) En los fragmentos analizados de La Misión hemos logrado que esa sensación de cansancio por saturación sea mucho menor y, como consecuencia, se llegue a comprender más fácilmente la trama. Aunque no hemos podido conseguir que los valores de la AD estén en la categoría Compensada.

d) Los valores de los porcentajes, salvo la V.A. ${ }_{1}$ de la UA nº 26, están dentro en la categoría Normal.

e) En el futuro pensamos seguir analizando las categorías del patrón rítmico y patrón pausativo, utilizando un mayor número de películas audiodescritas.

f) Existe una necesidad de abrir líneas de investigación que estudien las posibles variaciones, si las hubiera, en los usos de la velocidad y del ritmo, ya sea la voz de la $\mathrm{AD}$ masculina o femenina.

g) El audiodescriptor utiliza, en ciertos casos, la pausa interna con una intención discursiva estratégica para focalizar conceptos o expresiones relevantes que la persona ciega o con deficiencia visual debe considerar adecuadamente para comprender más fácilmente la trama. 


\section{BIBLIOGRAFÍA}

AENOR (2005): Norma UNE 153.020: Audiodescripción para personas con discapacidad visual. Requisitos para la audiodescripción y elaboración de audioguías, Madrid, AENOR.

Arcos Urrutia, J. M. (2012): “Análisis de guiones audiodescritos y propuestas para la mejora de la Norma UNE 153020", Tonos Digital, 22, accesible en: <http://www.tonosdigital.es/ojs/index.php/tonos/article/view/733/509>.

BenECKe, B. (2004): “Audio-Description”, Meta, 49 (1), págs. 78-80.

BeNECKe, B. y Dosch, E. (2004): Wenn aus Bildern Worte werden, Munich, Bayerischer Rundfunk.

BLAIR, A. (2010): "Information overload, then and now", The Chronicle Review, accesible en: <http://chronicle.com/article/Information-Overload-Then-and/ $125479 />$.

Bourne, J. y Jiménez Hurtado, C. (2007): "From the visual to the verbal in two languages: a contrastive analysis of the audio description of The Hours in English and Spanish”, en J. Díaz Cintas, P. Orero y A. Ramael (eds.), Media for All. Subtitling for the Deaf, Audio Description and Sign Language, Amsterdam, Rodopi, págs. 175-188.

BRAUn, S. (2007): "Audio Description from a discourse perspective: a socially relevant framework for research and training", Linguistica Antverpiensia, NS6, págs. 357-369.

BRAUN, S. (2008): "Audiodescription research: state of the art and beyond", Translation Studies in the New Millennium, 6, págs. 14-30.

Cebrián Herreros, M. (1994): Información radiofónica. Mediación técnica, tratamiento y programación, Madrid, Síntesis.

Cejudo Pinillos, M. (1991): "Museo tiflológico y sonocine, dos proyectos de la ONCE”, Integración, 5, págs. 26-28.

Chávez Miranda, J. R. (2006): "Ruido: efectos sobre la salud y criterio de su evaluación al interior de recintos”, Ciencia \& Trabajo, 8 (20), págs. 42-46.

Doloughan, F. y Rogers, M. (2005): "To see things and texts: audiovisual translation, audiodescription and multimodality", Paper delivered at conference on Multimodal Texts: Engaging Sign Systems, Oct. 22-23, at the University of Portsmouth.

Ellis, R. y McClintock, A. (1993): Teoría y práctica de la comunicación humana, Barcelona, Paidós.

Enoch, J., Campos, E. y Bedell, H. (1979): "Visual Resolution in a Patient Exhibiting a visual Fatigue or Saturation-like Effect. Probable Multiple Sclerosis”, Arch Ophthalmol, 97 (1), págs. 76-78.

FrazIER, G. (1975): The Autobiography of Miss Jane Pitman: An all-audio adaptation of the teleplay for the blind and visually handicaped, Film and Communication, San Francisco, San Francisco State University. 
Galisteo, J. R. (1994): “Cine para ciegos”, El Mundo, Comunicación, 6-211, pág. 5.

Greening, J. et al. (2010): A Comparative Study of Audio Description Guidelines Prevalent in Different Countries, London, RNIB Media and Culture Department.

Grice, H. P. (1975): "Lógica y conversación”, en L. M. Valdés Villanueva (ed.), La búsqueda del significado. Lecturas de filosofía del lenguaje, Madrid, Tecnos, págs. 511-530.

Hernández Bartolomé, A. y Mendiluce Cabrera, G. (2004): “Audesc: translating images into words for Spanish visually impaired people”, Meta, 49 (2), págs. 264-277.

HiLls, G. (1987): Los informativos en radiotelevisión, Barcelona, Paidós.

Hyks, V. (2005): "Audio Description and Translation. Two related but different skills”, Translation Today, 4, págs. 6-8.

ITAMI, H. (1977): "The concept of the amount of information in information evaluation”, Hitotsubashi Journal of Commerce and Management, 12 (1), págs. 33-43.

JimÉNEZ HuRTADO, C. (2007a): "De imágenes a palabras: la audiodescripción como una nueva modalidad de traducción y de representación del conocimiento", en G. Wotjak (ed.), Quo vadis Translatologie?, Leipzig, Frank und Timme, págs. 143-160.

JimÉnez Hurtado, C. (2007b): "Una gramática local del guión audiodescrito. Desde la semántica a la pragmática de un nuevo tipo de traducción”, en C. Jiménez Hurtado (ed.), Traducción y accesibilidad. Subtitulación para Sordos y audiodescripción para ciegos: nuevas modalidades de Traducción Audiovisual, Frankfurt, Peter Lang, págs. 55-80.

Jiménez Hurtado, C. (2010): "Un corpus de cine. Fundamentos teóricos de la audiodescripción”, en C. Jiménez Hurtado et al. (eds.), Un corpus de cine. Teoría y práctica de la audiodescripción, Granada, Tragacanto, págs. 13-56.

KNAPP, M. L. (1982): La comunicación no verbal, Barcelona, Paidós.

Kosslyn, S. (1994): Image and brain: the resolution of the imagery debate, Cambridge, MIT Press.

Kosslyn, S. (2005): "Mental images and the brain”, Cognitive Neuropsychology, 22 (314), págs. 333-347.

Magariños de Morentin, J. A. (2001): La(s) semiótica(s) de la imagen visual. Cuaderno de la Facultad de Humanidades y Ciencias Sociales, San Salvador de Jujuy $\mathrm{n}^{\mathrm{o}} 17$, Universidad Nacional de Jujuy.

MCLEISH, R. (1978): Técnicas de creación y realización en radio, Madrid, IORTV.

Navarrete Moreno, F. J. (1997a): "Aplicación al teatro del sistema AUDESC", Integración, 24, págs. 26-29.

Navarrete Moreno, F. J. (1997b): "Sistema AUDESC: el arte de hablar en imágenes”, Integración, 23, págs. 70-75.

Neetens, A., Lowenthal, A. y Martin, J. J. (1984): The visual system in Myelin Disorders, The Hague, Junk Publishers.

Ofcom (2000): ITC Guidance on Standards on Audio Description, accesible en: $<$ http://www.ofcom.org.uk/static/archive/itc/itc_publications/codes_guid ance/audio_description/index.asp.html>. 
Orero, P. (2005): "Audio Description: Professional Recognition, Practice and Standards in Spain”, Translation Watch Quarterly, 1, págs. 7-18.

Orero, P. (2007): "Sampling audio description in Europe”, en J. Díaz Cintas, J. Orero y A. Ramael (eds.), Media for All. Subtitling for the Deaf, Audio Description and Sign Language, Amsterdam, Rodopi, págs. 111-125.

Orero, P. y Vilaró, A. (2012): "Eye tracking analysis of minor details in films for audio description", MonTI, 4, págs. 295-319.

Piety, P. (2004): "The language system of audio description: an investigation as a discursive process", $J V I B, 98$ (8), págs. 453-469.

Puigdomènech, L. et al. (2010): "Audio description of films: state of the art and protocol proposal”, en L. Bogucki y K. Kredens (eds.), Perspectives on Audiovisual Translation, Frankfurt am Main, Peter Lang, págs. 27-43.

Ramael, A. y Vercautern, G. (2007): "Audio describing the exposition phase of films. Teaching students what to choose”, Trans, 11, págs. 73-93.

RAMAel, A. y Vercautern, G. (2010): "The translation of recorded audio description from English into Dutch", Perspectives: Studies in Translatology, 18 (3), págs. $155-171$.

Real Academia Española (2001): Diccionario de la lengua española, Madrid, Espasa.

Recanati, F. (2004): Literal Meaning, Cambridge, Cambridge University Press.

Recanati, F. (2007): Perspectival Thought: A Plea for (Moderate) Relativism, Oxford, Clarendon Press.

Regan, D. y Neima, D. (1984): "Visual fatigue and visual evoked potentials in multiple sclerosis, glaucoma, ocular hypertension and Parkinson's disease”, Journal of Neurology, Neurosurgery and Psychiatry, 47, pags. 673-678.

RosEnBERG, D. (2003): "Early modern information overload", Journal of the History of Ideas, 64 (1), págs. 1-9.

SAlway, A. y Palmer A. (2007): "Describing actions and thoughts", Paper delivered at the Advanced Seminar: Audiodescription - towards an interdisciplinary research agenda. University of Surrey, 28-29 June.

SAlwaY, A. (2007): "A Corpus-based analysis of the language of Audio Description”, en J. Díaz Cintas, P. Orero y A. Ramael (eds.), Media for All. Subtitling for the Deaf, Audio Description and Sign Language, Amsterdam, Rodopi, págs. 151-174.

Sedlack, R., Showm, B. y Keller, K. (2009): Graphics and visual communication for managers, Mason, South-Western Educational Publishing.

Silverwood, A. (1992): “Audiodescription”, DAM (Disability Arts Magazine), 2-4, págs. 10-14.

SNYDER, J. (2005): "Audio Description. The visual made verbal across arts disciplines -across the globe”, Translating Today, 4, págs. 15-17.

SPERBER, D. y WiLson, D. (1994): La relevancia: comunicación y procesos cognitivos, Madrid, Visor.

STANLEY, J. (2000): "Context and logical form”, Linguistics and Philosophy, 23 (4), págs. 391-434.

Stanley, J. (2007): Language in Context: Selected Essays, Oxford, Oxford University Press. 
Van Dijk, T. A. (2008): Discourse and context. A sociocognitive approach, Cambridge, Cambridge University Press.

VÁzQuez MARTín, A. (2008): "El mercado potencial de la accesibilidad: nuevos consumidores”, en C. Jiménez Hurtado y A. Rodríguez Domínguez (eds.), Accesibilidad a los medios audiovisuales para personas con discapacidad (AMADIS'O7), Madrid, Real Patronato sobre Discapacidad, págs. 37-42.

Vercauteren, G. (2007): "Towards a European Guidelines for Audio Description”, en J. Díaz Cintas, P. Orero y A. Ramael (eds.), Media for All. Subtitling for the Deaf, Audio Description and Sign Language, Amsterdam, Rodopi, págs. 139-150.

Vercauteren, G. (2012): "A narratological approach to content selection in audio description. Towards a strategy for the description of narratological time", MonTI, 4, págs. 207-231.

WhiteheAd, J. (2005): "What is audio description", International Congress Series, 1282, págs. 960-963.

Whitelaw, S. (2008): "Health information: a case of saturation or 57 channels and nothing on?", The Journal of the Royal Society for the Promotion of Health, 128 (4), págs. 175-180.

WiLdberger, H. y Robert, Y. (1988): "Visual fatigue during prolonged visual field testing in optic neuropathies”, Neuro-Ophthalmology, 8 (4), págs. 167-174. 